\title{
January 2014 Critical Care Journal Club
}

\begin{abstract}
Kamps MJ, Horn J, Oddo M, Fugate JE, Storm C, Cronberg T, Wijman CA, Wu O, Binnekade JM, Hoedemaekers CW. Prognostication of neurologic outcome in cardiac arrest patients after mild therapeutic hypothermia: a meta-analysis of the current literature. Intensive Care Med. 2013;39(10):1671-82. [CrossRef] [PubMed]
\end{abstract}

Cohort studies performed prior to the advent of therapeutic hypothermia had shown that severe deficits in motor response to painful stimuli, or deficits in certain cranial nerve reflexes, could be used to rule-out the possibility of meaningful neurological recovery in patients who did not regain consciousness within 48-72 hours after cardiac arrest. However, subsequent reports have challenged the reliability of these findings in patients who received therapeutic hypothermia - some of whom recovered despite grim neurological findings.

These authors performed a meta-analysis to determine whether neurological findings performed 72 hours after cardiac arrest (of all causes) could be used to prognosticate neurological outcome in patients who received mild therapeutic hypothermia. They identified ten studies with a cumulative sample size of 1153 patients, and used this data to calculate the sensitivity and false positive rate for four neurological findings. A poor neurological outcome was somewhat ill-defined, as included studies used different neurological scoring systems, and periods of follow-up varied, but generally would equate to an outcome no better than a vegetative state. The following operating characteristics were calculated:

\begin{tabular}{|l|l|c|}
\hline & \multicolumn{1}{|c|}{ False Positive Rate } & \multicolumn{1}{c|}{ Sensitivity } \\
\hline $\begin{array}{l}\text { GCS motor score }<3 \\
(\mathrm{n}=811)\end{array}$ & $0.21(95 \% \mathrm{Cl} 0.08-0.43)$ & $0.80(95 \% \mathrm{Cl} 0.63-0.91)$ \\
\hline $\begin{array}{l}\text { Absent pupillary reflex } \\
(\mathrm{n}=566)\end{array}$ & $0.004(95 \% \mathrm{Cl} 0.001-0.03)$ & $0.22(95 \% \mathrm{Cl} 0.18-0.27)$ \\
\hline $\begin{array}{l}\text { Absent corneal reflex } \\
(\mathrm{n}=429)\end{array}$ & $0.02(95 \% \mathrm{Cl} 0.002-0.13)$ & $0.32(95 \% \mathrm{Cl} 0.27-0.39)$ \\
\hline $\begin{array}{l}\text { Bilaterally absent } \mathrm{N} 20 \\
\text { somatosensory } \\
\text { evoked response } \\
(\mathrm{n}=492)\end{array}$ & $0.007(95 \% \mathrm{Cl} 0.001-$ & $0.50(95 \% \mathrm{Cl} 0.42-0.57)$ \\
\hline
\end{tabular}

This study probably represents the best current data to support decisions regarding withdrawal of life support in patients who received therapeutic hypothermia post cardiac arrest. A very low false positive rate is necessary to avoid recommending withdrawal of support from a patient who might recover. Only absent pupillary response and absent N20 somatosensory evoked response meet this requirement. Unfortunately, the sensitivity of these two tests are both poor, so we might expect that many will suffer 
prolonged life support to no good end if these tests are all we have to go on. It would make the most sense clinically to look at the pupillary reflex first, then order the SSEP if the pupils react, but we have no information of the sensitivity of the tests used in series. The findings of this study will introduce more uncertainty into certain end-of-life discussions. It has been posited that hypothermia might have confounded neurological prognostication simply by delaying clearance of sedation drugs. If that is the case, future studies may show improved performance of clinical prognosticators by giving the patient more time to recover - perhaps waiting 72 hours after normothermia is achieved instead of 72 hours after the arrest. Until further studies are available though, we will have to proceed as best we can in patients who do not awaken within 72 hours after cardiac arrest, taking into account the entire clinical status of each individual patient. Younger patients with less comorbidity seem to have better recuperative capability. Studies have shown that patients who suffer cardiac arrest secondary to sepsis or cancer have a very low chance of survival to discharge regardless of their neurological findings.

\section{Nielsen N, Wetterslev J, Cronberg T, et al. Targeted temperature management at $33^{\circ} \mathrm{C}$ versus $36^{\circ} \mathrm{C}$ after cardiac arrest. N Engl J Med. 2013;369(23):2197-206. [CrossRef] [PubMed]}

Two previous randomized controlled trials have shown that patients who do not rapidly recover consciousness after cardiac arrest from ventricular fibrillation or ventricular tachycardia, have improved neurological outcomes if they receive 24 hours of therapeutic hypothermia in the range of 32-34C. These authors set out to confirm this finding, and better define the optimal therapeutic temperature.

They randomized 939 patients who remained unconscious after out-or-hospital cardiac arrest to temperature management with a goal temperature of $33 \mathrm{C}$ versus $36 \mathrm{C}$. Life support was discontinued during the first week in 247 patients based on neurological findings associated with poor outcomes. These included brain death, status myoclonus, or a motor GCS score of two or less plus either status epilepticus or absent cortical N20 somatosensory evoked potential. Mortality at the end of the study was 50\% vs. $48 \%$ $(p=0.51)$. At 180-day follow-up, the percentage of patients who died or had poor neurological outcome was $54 \%$ vs. $52 \%(p=0.78)$.

This study was well designed, and larger than both previous randomized controlled trials combined. It was well powered to detect a $20 \%$ reduction in the hazard ratio for death. 180-day follow-up was accomplished for all survivors. It seems to be the most convincing data available related to post-arrest hypothermia, but interpretation is debatable. If the results are valid, it could mean that temperature control with a target of $36 \mathrm{C}$ is equally beneficial to temperature control with a target of 33C. This would support continuation of our current practice of careful temperature management, with perhaps less danger of potential complications of hypothermia and less need for sedation to control shivering. Alternatively, it could mean that therapeutic hypothermia doesn't work after all. The later interpretation might be consistent with a recent large RCT that showed that starting hypothermia therapy in the prehospital setting was not clinically beneficial in patients who suffered cardiac arrest, even though it significantly 
reduced the time it took to achieve a temperature of 34C. (JAMA 2014;311:45-52). I think the first interpretation is more likely valid, and would recommend that we target $36 \mathrm{C}$ based on the findings of this study. Therapeutic hypothermia is a labor-intensive and potentially dangerous therapy. We have seen fatal hypothermia result from poor temperature control during a course of "therapeutic hypothermia". As with almost everything in our field, "less is more".

\section{Slutsky AS, Ranieri VM. Ventilator-induced lung injury. N Engl J Med. 2013 Nov 28;369(22):2126-36. [CrossRef] [PubMed]}

This paper did not address pulmonary complications of mechanical ventilation such as oxygen toxicity, or barotrauma, but it was a good overall review of ventilator associated lung injury (VALI). The discussion of the interplay of ventilator pressures and volumes was particularly helpful. The main point here is that alveolar overdistention is likely the main mechanism of VALI. Elevated transpulmonary pressure is closely related to lung overdistention, but it cannot be directly measured in any clinically practical way. Transpulmonary pressure equals the alveolar pressure (plateau pressure) minus the pleural pressure. We often use the alveolar pressure as a surrogate for transpulmonary pressure, but this can be misleading. A morbidly obese patient on mechanical ventilation may require high alveolar pressure to inflate their heavy chest wall, without experiencing elevated transpulmonary pressures or overdistention. Another patient in respiratory distress on a mechanical ventilator may have modest positive alveolar pressures, yet generate very high negative pleural pressures in their effort to pull breaths through the ventilator - this could lead to elevated transpulmonary pressures and alveolar overdistention. Therefore even though alveolar pressure (plateau pressure) was used in the ARDSnet low-tidal-volume RCT, it is not always an accurate measure of the risk for VALI. Read the article for more details.

\section{Cuenca AG, Gentile LF, Lopez MC, et al. Development of a genomic metric that can be rapidly used to predict clinical outcome in severely injured trauma patients. Crit Care Med. 2013;41(5):1175-85. [CrossRef] [PubMed]}

This was a fantastic research question buried in a very hard-to-read paper with a weak study design. The authors analyzed genomic data from a retrospective cohort of 167 patients who suffered severe trauma, and identified a panel of 63 leukocyte genes that distinguished between patients who had good and poor recoveries. These were collected into a genomic panel that was then re-analyzed using a different laboratory method, and reduced to a single prognostic metric. Although the authors call this stage of the study "validation", it is not - since the same patients were simply retested for the same set of genes. Still, the receiver operating characteristic curves shown in Figure 4 on page 1183 of the article are fascinating. They show that APACHE II severity adjustment is almost without value in discriminating a complicated recovery in this set of patients, with an area-under-the-curve of 0.59 (a coin-toss would have an AUC of 0.50). The genomic models had an AUC as high as 0.81 . The concept that we might be able to measure the response of our leukocytes to systemic injury, and that this response might be more predictive of outcomes than all the clinical elements of the APACHE II 
score is highly thought-provoking. Hope to see a true validation study of this technology soon, using an independent sample of patients.

Bernal W, Wendon J. Acute liver failure. N Engl J Med. 2013 Dec 26;369(26):252534. [CrossRef] [PubMed]

We have been interested in this topic for many years in relation to our liver transplant program, and our fellows and staff have previously developed and published a neuroprotective clinical protocol for the prevention and treatment of cerebral edema in patients with acute liver failure - the leading cause of death in ALF (1). But we were disappointed in this review. The authors offer only a brief treatment of the most controversial aspect of patient care - the use of intracranial pressure monitors. The authors "monitor intracranial pressure only in patients with clinical signs or evidence of developing intracranial hypertension", but do not discuss specifically what those are. In fact, some clinical signs of intracranial hypertension, such as papilledema and Cushing reflex are notoriously insensitive for detecting the development of intracranial hypertension. In our opinion, the most sensitive and practical clinical sign to detect intracranial hypertension is the development of grade III or IV encephalopathy. The controversy regarding whether or how to best measure ICP is largely ignored in this article. No mention is made of many important aspects of practical bedside management that are vital to supporting a patient through a period of intracranial hypertension. For instance, no mention is made of the calculation and management of cerebral perfusion pressure (CPP) related to ICP monitoring. [CPP=mean arterial pressure - ICP]. It is important to maintain adequate CPP (typically $>60 \mathrm{mmHg}$ ) during periods of intracranial hypertension in order to prevent ischemic brain injury. We have also found head position to be strikingly important in patients with cerebral edema. Patients with ICP monitors who are laid flat for central line placement often suffer immediate, potentially life-threatening increases in ICP. This likely explains why ALF patients sometimes suffer brain injury during transport, or other situations in which their head may be lowered. Prognostication is key to the decision to transplant the patient, or transport the patient to a transplant center - but the authors only address the topic in general terms, without actually providing any quantitative prognostic criteria or any specific operating characteristics of any of the competing prognostic scoring systems. This review is inadequate for a critical care physician who actually provides bedside care of patients with acute liver failure.

Robert Raschke, MD MS

Associate Editor

\section{References}

1. Raschke RA, Curry SC, Rempe S, Gerkin R, Little E, Manch R, Wong M, Ramos A, Leibowitz Al. Results of a protocol for the management of patients with fulminant liver failure. Crit Care Med. 2008;36(8):2244-8. [CrossRef] [PubMed] 